\title{
A SMART AUTOMOTIVE ACCELEROMETER WITH ON-CHIP AIRBAG DEPLOYMENT CIRCUITS
}

\author{
Leland "Chip" Spangler Christopher J. Kemp \\ Ford Microelectronics, Inc. \\ 9965 Federal Drive \\ Colorado Springs, CO 80921
}

\begin{abstract}
This paper describes a "smart" accelerometer for use in automotive passive restraint systems. This accelerometer performs, in a single integrated component, all of the sensing and signal processing functions required to assess vehicle crash severity and generate a timely airbag deployment signal if and when it is needed.
\end{abstract}

\section{INTRODUCTION}

Automolive passive restraint systems continue to utilize the benefits of microelectronics to provide more sophisticated occupant safety features. Traditional microelectronic advantages such as miniturization, system integration, and part count reduction are being used to provide additional passenger safety through wider applications and more advanced features.

Sensors play a primary role in these smart systems as they provide the information the system uses to make deployment decisions. Occupant detection and occupant position sensors will be used in the future to allow these features to be implemented so that occupant safety in a crash can be improved. Accelerometers are, however, the first high volume electronic sensor to see widespread use in electronic-based passive restraint systems. Already the evolution of electronic frontal airbag systems is placing increasing demands on accelerometers, including greater accuracy, better diagnostics, improved robustness, smaller size, and lower cost. The ever decreasing size of electronic components, with the ability to integrate more functions, will drive the components to incorporate more and more of the system function.

This paper describes a "smart" automotive accelerometer that continuously evaluates vehicle decelerations and generates a timely airbag deployment command if needed. Vehicle and system level requirements have led to the adoption of several key driving factors in designing this version of the accelerometer. These requirements include a high degree of accuracy in accelerometer sensitivity and offset, insensitivity to temperature as well as voltage and process variations, a high level of reliability, low sensitivity to electromagnetic radiation and power supply noise, and low power consumption. The specification of low quiescent current of this fully-integrated system supports key-off operation as well as the low power requirements of electric and hybrid passenger vehicles. In addition, the small size supports the demand for reduced module size which in tum facilitates vehicle-level installation.

These factors lead to a different approach to system partitioning that supports the requirements of next generation frontal and side passive restraint systems. The partitioning reduces demands on the system and microprocessor by performing signal processing and algorithm computation in the accelerometer so that the output of the accelerometer is a signal that is used to activate the passive restraint.

\section{APPLICATION}

Passive restraint systems are refered to as "distributed" when mechanical acceleration switches are used at multiple positions near the front of the vehicle to evaluate crash severity and decide "if and when" an airbag should be deployed (Figure 1). A separate wiring hamess is used to connect these under-hood sensors with the central diagnostic module. Single-point electronic passive restraint systems (Figure 1) eliminate the under-hood sensors and wiring harness, and instead use a single accelerometer to provide data for a microprocessor-based algorithm that makes deployment decisions. The electronic.module that contains the accelerometer and microprocessor is located in the passenger compartment of the vehicle. This simplifies vehicle assembly, lowers cost, and improves reliability while offering system and component self-test capability.

\section{SENSOR DESIGN}

The accelerometer is a two-chip device that consists of a differential, capacitive micromachined sense element die assembled in an IC package along with a CMOS interface chip containing EEPROM calibration circuits. It is an adaptation of the accelerometer reported by Cole [1] and an extension of the accelerometer we previously reported [2]. The system block diagram is shown in Figure 2, where the signal path is indicated by the bold arrows. The three modular components of the accelerometer (two chips and the package) can be developed, scaled, or optimized without making changes in the other components of the sensor. This flexibility offers advantages in lowering development costs and time, while allowing application specific designs that meet specific system specifications.

The sense element die produces capacitance variations in response to the acceleration signal. The sense element chip uses a

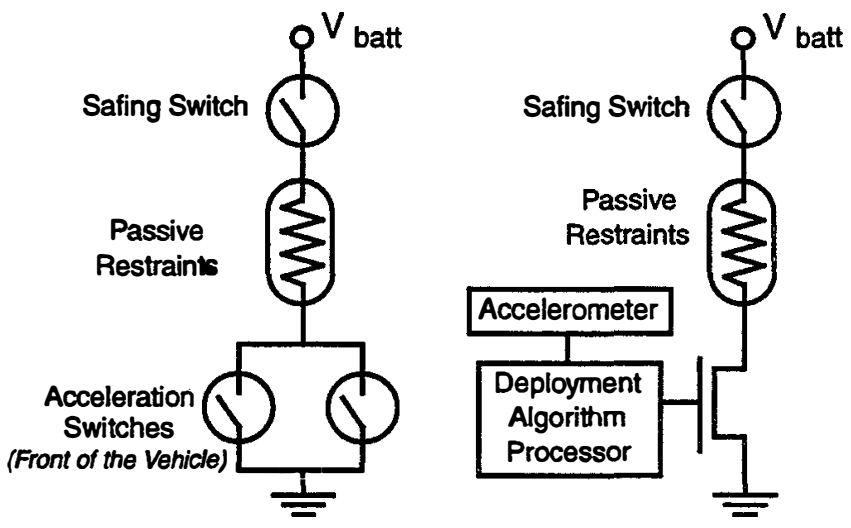

Figure 1. Schematics of automotive passive restraint systems. On the left is a distributed sensor system, on the right is a single-point sensing system. 


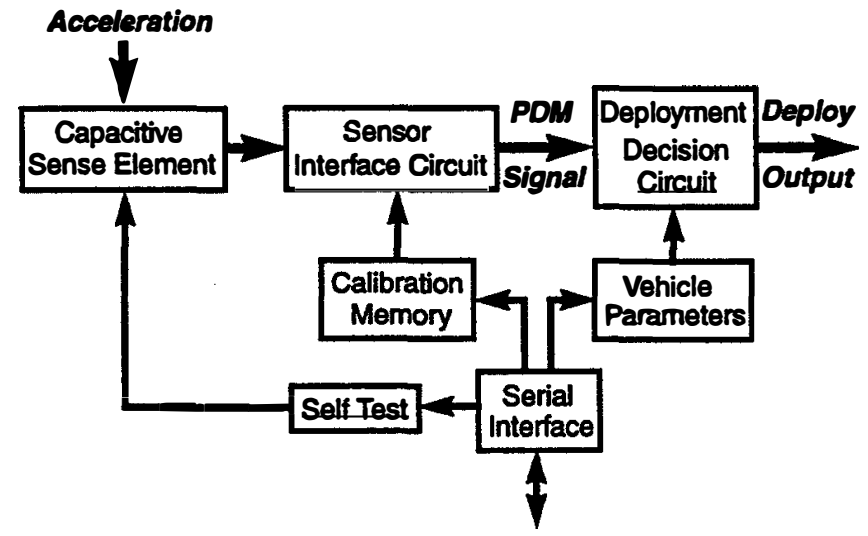

Figure 2. The block diagram of the smart accelerometer.

mirror-symmetric arrangement of two torsional microstructures that generate a fully differential, femtofarad-level signal that is fod to the interface chip via bond wires.

The interface chip uses a charge-mode delta-sigma modulator to convert the sense element signals into a pulse-density-modulated output that is proportional to the applied acceleration[3]. The interface chip has EEPROM and a resistor string DAC that allow the gain and offset of the completed device to be trimmed to the desired specification. A programmable digital algorithm uses circuit blocks that calculate magnitudes, changes in velocity over ime intervals, and peak acceleration data to make deployment decisions. The programmable nature of this algorithm allows it to be configured for many vehicle types. A serial interface allows the device to communicate with a microprocessor to facilitate accelerometer calibration and algorithm programming during device manufacture, and to facilitate data transfer and a secure self test activation after the device is installed in a vehicle.

This device is presently packaged in a multilayer ceramic package, but the modular nature of the technology allows other versions of the accelerometer to be packaged in a conventional plastic integrated circuit package [2].

\section{MICROMACHINED SENSE ELEMENT}

The sense element contains two torsional microstructures in which movable capacitor plates are suspended, from a central "pedestal" support, above fixed capacitor plates metallized on a glass substrate. A simplified schematic is shown in Figure 3. Typical values for the capacitance at zero acceleration are $150 \mathrm{fF}$ while full-scale acceleration results in approximately a $15 \mathrm{fF}$ increase to $C_{A}$ and $C_{D}$ and a somewhat smaller decrease to $C_{B}$ and $\mathrm{C}_{\mathrm{C}}$.

The element is fabricated using a single-crystal silicon, dissolved-wafer process [2] on a glass substrate which is mounted in the same package with, and wirebonded to, a CMOS ASIC which contains all of the remaining circuitry. Dimensioning of the element's plate thickness, air gap, torsion beams, and damping holes creates an overdamped, second-order mechanical system with a resonant frequency which is typically $2.4 \mathrm{KHz}$ and a dominant pole which is typically at $375 \mathrm{~Hz}$. This bandlimiting of the acceleration signal by the sense element is used to provide antialias filtering for the sampled-data interface circuit and signal filtering for accurate algorithm processing. To provide a self test capability, the sense element's glass substrate incorporates additional metallized regions which are used, during a self test operation, to electrostatically deflect the movable plates.

The central pedestal support greatly reduces the susceptibility of the sensor to die stresses since it mechanically isolates the microstructure from the substrate and from the packaging materials. This eases packaging constraints and also improves the manufacturability of the sensor. This mechanical configuration is one factor that allows the accelerometer to be manufactured without temperature compensation circuitry. The use of two torsional microstructures in the accelerometer offers several advantages that are a result of the four capacitors being configured as a full bridge. This electrical and mechanical symmetry of the structure greatly reduces sensitivity to cross axis forces, power supply noise, and electromagnetic radiation, through the use of a fully-differential signal path.

The design of the torsion arms' dimensions offers flexibility in meeting a variety of performance specifications that the applications require. Typical torsion arm lengths are in the range of 250 micrometers long with a cross sectional area that is in the range of 100 square micrometers. The "heavy" side of the microstructure is approximately 3 times more massive than the "light" side, and each suspended microstructure occupies an area of approximately 0.8 square millimeters.

The fixed plates of the sense element are formed from metalized regions on the glass substrate. These fixed plates are larger than the silicon microstructure so that cross axis forces causing the microstructure to twist parallel to the substrate do not cause the nominal capacitance to change. The electrostatic self test is implemented on the "heavy" side of the microstructure by placing additional fixed electrodes under the movable silicon. Self test voltages, provided by the interface chip, are applied to these fixed plates during the self test operation, and the resultant electrostatic force causes the microstructure to rotate in a manner that mimics the microstructure's motion during a vehicle crash.

The initial configuration of the device uses a hermetic package and a sense element die that is not capped. With this configuration, electrical contact to the microstructure is made via a bond pad and wire that are directly applied to the pedestal region. The hermetic seal of the ceramic package results in a fixed gaseous environment that is an important factor in determining the mechanical response of the microstructure to vibrations. Gas viscosity changes as a function of temperature and this changes the damping characteristics of the microstructure. Both gas type and pressure must also be considered as factors that influence the mechanical response of the microstructure. The pressure inside the package is dependent primarily on two factors: the sealing temperature of the lid to the package, and the ambient pressure at sealing. The lid is solder sealed to the ceramic package using an

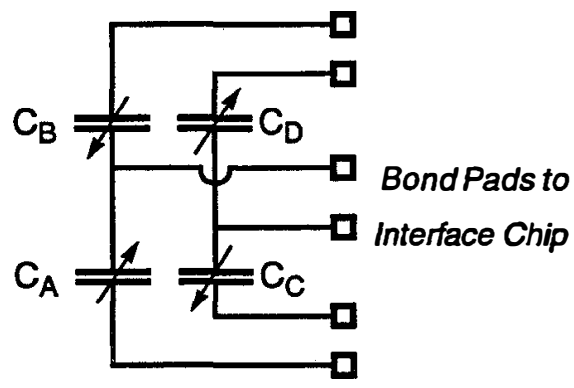

Figure 3. Schematic of the differential capacitive sense element. 


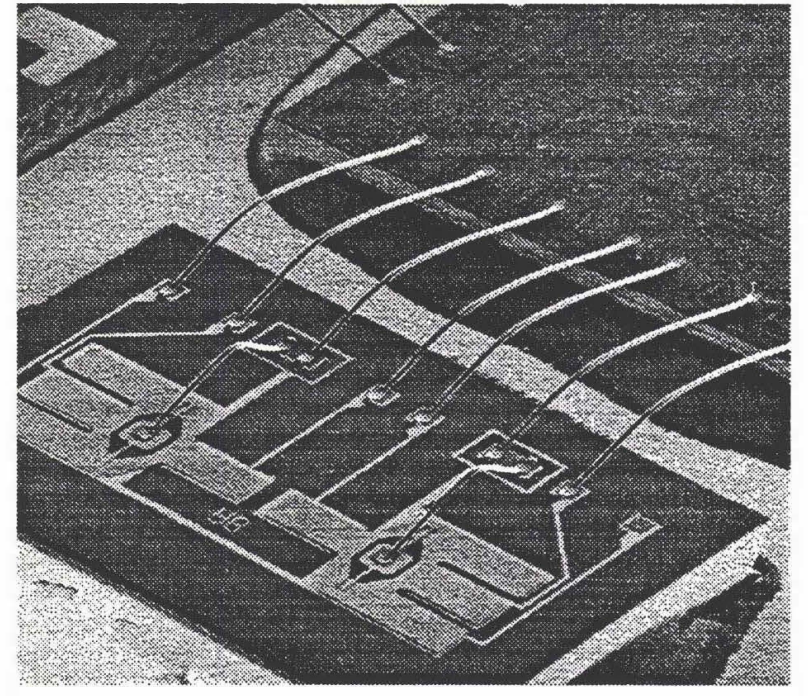

Figure 4. Scanning electron micrograph of the sense element chip wire bonded to the interface chip in a ceramic package

elevated temperature in a conventional furnace. Colorado Springs is located at an altitude of over 6500 feet which results in a local barometric pressure that is about $80 \%$ of that at sea level. The result is that the pressure in the cap is less than 0.5 atmospheres.

\section{INTERFACE AND ALGORITHM CIRCUITS}

The next modular component of the accelerometer to be discussed is a CMOS interface chip which has two primary functions. First, the interface circuit converts the differential femtofarad-level capacitance signals from the sense element to a pulse-density-modulated output. This circuit contains calibration circuitry to remove offset and sensitivity variation from the fully assembled device. The Deployment Decision Circuit (DDC) is the second major function on the interface chip. The DDC implements a programmable algorithm which, when given the appropriate parameters (based on the vehicle body and sensor location) decides if and when a passive restraint should be activated. In addition, the interface chip implements the self test and reset functions as well as the serial interface to both the system and to production test/calibration equipment. The interface chip, fabricated in a $1.6 \mathrm{~mm}$ CMOS process, has a die area of approximately $19 \mathrm{~mm}^{2}$.

The interface circuit is a first-order, charge-mode $\Delta \Sigma$ modulator similar to that reported in [3]. The primary difference is the fully differential nature of the present sensor and signal path. The interface circuit drives the acceleration-dependent sense element capacitors with voltage transitions which pump charge packets either onto, or off of, the modulator's integrator on each cycle of the $250 \mathrm{KHz}$ clock. On a given clock cycle, charge is pumped onto the integrator if its output voltage on the previous clock cycle was negative, and off of the integrator if its output voltage on the previous clock cycle was positive. The output pulse density is determined by the ratio of the charge pumped on to the charge pumped off. These charges depend on the sense element capacitances (and thus the acceleration) as well as the voltage transitions produced by the interface circuit. Calibration is performed by precise adjustment of these voltage transitions [3]. The interface circuit is designed to be very sensitive to changes in sense element capacitances; a change in the charge packet sizes of only six electrons produces a one hertz change in output pulse density.

The nature of the pulse density modulated (PDM) output simplifies the implementation of the on-chip deployment decision circuit, whose (simplified) block diagram is shown in Figure 5. Each pulse of PDM output corresponds to a specific velocity change, so for example, with a $50 \mathrm{~g}$ calibration and a $4 \mathrm{MHz}$ clock, each PDM pulse corresponds to a change velocity of approximately $2 \times 10^{-3}$ meters/sec. This approach allows the deployment algorithm circuits to be implemented, with a minimum amount of chip real estate, using counters, timers, and pulse integrators.

The "prediscriminator" in the DDC constantly evaluates the output of the accelerometer, and based on the programmed parameters and the acceleration, a "threshold" logic signal is generated. This threshold signal is used as an enable for the other blocks in the "discriminator" portion of the DDC.

The discriminator output is based not only on acceleration thresholds and magnitude calculations but also on total velocity and acceleration changes calculated over particular time periods. The DDC allows these calculations to be made simultaneously in real time. The output of these individual blocks are then logically combined to implement the overall deployment decision algorithm. Since the DDC's counters, timers, and pulse integrators are programmable over a wide range, the algorithm parameters can be adjusted to optimize deployment decision performance for a particular vehicle body style and sensor location.

\section{SENSOR PACKAGING}

The modular approach to the accelerometer is also evident in the packaging technologies selected for the device. The first samples of this accelerometer use conventional packaging technology in which the two die are mounted in the cavity of a multilayer ceramic DIP. The accelerometer is completed by wirebonding the two die together using conventional gold wire bonds. Wire bonds are also used to connect the interface chip to the package pins. A solder lid is attached over the package cavity as discussed previously.

The accelerometer package and die mounting influence the orientation of the primary axis of sensitivity. The ceramic through-hole package provides orientation flexibility while at the same time the solder sealed lid ensures a controlled ambient for the accelerometer. A SIP style package has also been developed and this allows the axis of sensitivity to be parallel the module printed wiring board.

While the multilayer ceramic technology has been used to provide a controlled and reliable environment for the microstructure, the hermetic packaging requirement can also be transferred to the sense element die through the development of a wafer-level capping technology that is compatible with the dissolved wafer process [2]. Wafer level capping allows the accelerometer to also be assembled in a conventional molded plastic

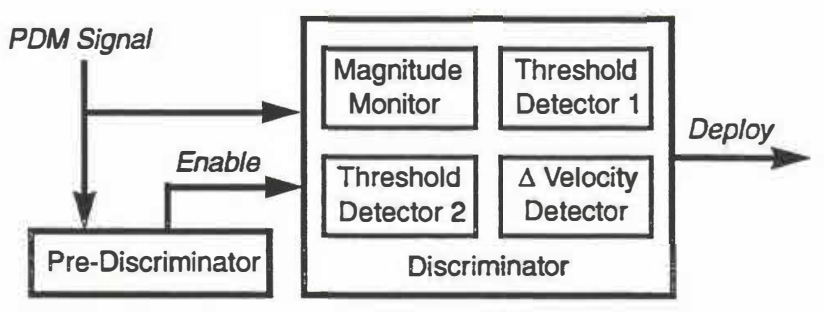

Figure 5. The block diagram of the deployment decision circuit. 


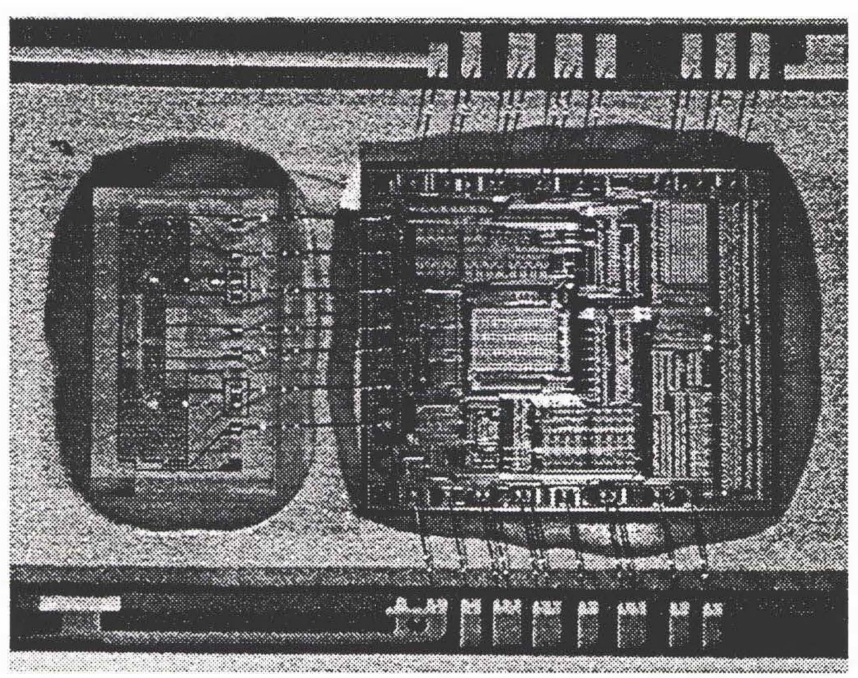

Figure 6. Photograph of the two-chip smart accelerometer.

package which supports many package styles and lead configurations while lowering overall sensor cost.

\section{SUMMARY AND RESULTS}

The accelerometer and its modular components have been fabricated with technologies that will continue to evolve to support a variety of sensor configurations, cost reductions, and feature optimizations. The configuration discussed in this paper offers excellent accelerometer performance, reliability, and manufacturability, as well as design features that minimize overall system costs. Figure 6 is a photomicrograph of the accelerometer, showing the sense element die and the signal processing ASIC in the ceramic package with the lid removed.

The sensor is designed to operate from a 5 volt supply and an extemal $4 \mathrm{MHz}$ clock. On-chip dividers generate an interleaved two phase clock at $250 \mathrm{KHz}$ which is the sampling frequency for the delta-sigma modulator. Greater clock speeds can be used to increase
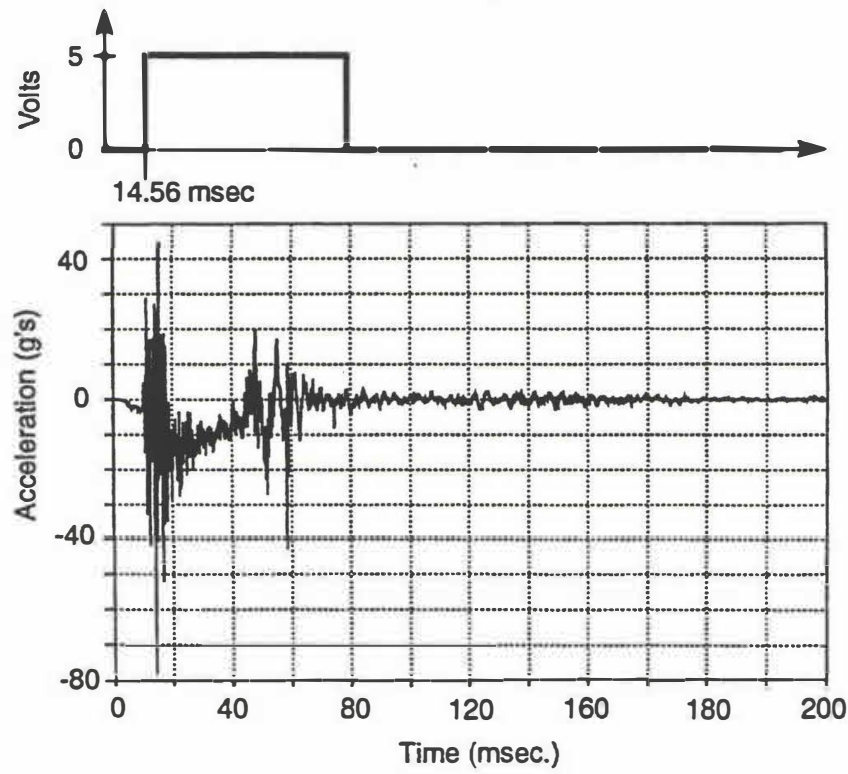

Figure 7. Acceleration data recorded at the crash barrier in a 14 mph impact with a $90^{\circ}$ wall (bottom) and the measured output of the smart accelerometer (top). the resolution of the sensor. The part has been tested to clock frequencies up to $16 \mathrm{MHz}$.

The accelerometer is typically calibrated under specific acceleration conditions in a custom designed centrifuge or "shaker." These calibration instruments use reference devices to monitor the acceleration applied to each device that is being calibrated. Only two room temperature readpoints (0g's and 15g's) are used for calibration, even though the full scale range of the accelerometer is $+/-50 \mathrm{~g}$ 's. The exact reference value measured at these readpoints is then used by the test software to generate specific calibration coefficients for each device. These parameters are then programmed into the accelerometer's EEPROM to set the offset and sensitivity of the accelerometer. Sensitivity and offset have temperature coefficients of less than $60 \mathrm{ppm} /{ }^{\circ} \mathrm{C}$ at the worst case temperature and voltage conditions.

Measured performance of the integrated airbag deployment system, for a particular crash, is depicted in Figure 7. The crash acceleration waveform is shown along with the deploy pulse, which occurred at $14.56 \mathrm{msec}$ after impact $(t=0)$. This differs by only 740 $\mu \mathrm{sec}$ from the simulated deploy pulse which occurred at $15.30 \mathrm{msec}$ after impact. A wide variety of responses is possible from the device based on accelerometer calibration and DDC algorithm coefficients.

The accelerometer has been subjected to standard automotive integrated circuit reliability tests. This includes temperature cycling, vibration endurance, shock and drop testing, and accelerated life testing. Large quantities of parts have been exposed to these tests and the successful results demonstrate the sensor's ability to withstand the automotive environment. Module and vehicle-level testing of the accelerometer at the crash barrier along with real world tests in fleet vehicles has also proven that the design objectives have been met.

\section{ACKNOWLEDGEMENT}

The authors would like to acknowledge the staff at Ford Microelectronics, Inc. who have worked to make this project successful. In addition, the support of the Automotive Components Division and Ford Research Laboratories has been essential.

\section{REFERENCES}

1. J. Cole, "A new sense element technology for accelerometer subsystems," Transducers '91, June 24-27, 1991, pp. 93-96.

2. L. Spangler, C. Kemp, “ ISAAC (Integrated Silicon Automotive Accelerometer)", 8th International Conference on Solid-State Sensors and Actuators, Transducers ' 95 , Stockholm, Sweden, June 25-29, 1995, pg. 585.

3. C. Kemp, L. Spangler, "An Accelerometer Interface Circuit," 1995 Custom IC Conference, May 1-4, 1995. 